\title{
Sensitivity of Phytophthora nicotianae var. parasitica Causing Buckeye Rot of Tomato to Commonly Used Fungicides in Himachal Pradesh
}

\author{
Gurpreet Kaur* and D.K. Banyal \\ Department of Plant Pathology, CSK Himachal Pradesh Krishi Vishvavidyalaya, \\ Palampur 176 062, Himachal Pradesh, India \\ *Corresponding author
}

\section{A B S T R A C T}

Buckeye rot caused by Phytophthora nicotianae var. parasitica (dastur) Waterhouse is the serious menace to the cultivation of tomato in Himachal Pradesh. Seventeen isolates of Phytophthora nicotianae var. parasiitca causing buckeye rot were collected from different districts of Himachal Pradesh. Sensitivity of these isolates to three commonly used fungicides i.e. mancozeb $75 \mathrm{WP}$

\section{Keywords}

Phytophthora nicotianae var. parasitica, Buckeye rot, Tomato, Sensitivity, Fungicides, Management

Article Info

Accepted:

10 July 2019

Available Online:

10 August 2019 (Indofil M-45), copper-oxychloride 50WP (Blitox-50) \& metalaxyl-M 4\%+ mancozeb 64\%WP (Ridomil Gold) in Himachal Pradesh was examined by dual culture technique. On the basis of sensitivity to fungicides the 17 isolates were categorized into three sensitivity classes i.e. highly sensitive, moderately sensitive and less sensitive isolates. Out of seventeen isolates, nine isolates were highly sensitive (Pn-3, Pn-4, Pn-5, Pn-7, Pn-9, Pn-10, Pn-12, Pn-14 \& Pn-17), seven isolates (Pn-1, Pn-2, Pn-6, Pn-8, Pn-11, Pn-15 \& Pn-16) were moderately sensitive and one isolate (Pn-13) was found less sensitive to mancozeb 75WP. Sensitivity to copper-oxychloride 50WP revealed that seven isolates (Pn-2, Pn-3, Pn-9, Pn-10, Pn-11, Pn-13 \& Pn-16) were highly sensitive, nine isolates (Pn-1, Pn-4, Pn-5, Pn-7, Pn-8, Pn-12, Pn-14, Pn-15 \& Pn-17) were moderately sensitive and one isolate (Pn-6) was found less sensitive to copper-oxychloride. Whereas, eleven isolates (Pn-2, Pn-3, Pn-4, Pn-5, Pn-6, Pn-10, Pn-11, Pn-12, Pn-13, Pn-15 \& Pn-17) were highly sensitive, five isolates were moderately sensitive (Pn-1, Pn-7, Pn-9, Pn-14 \& Pn-16) and only one isolate (Pn-8) was found less sensitive to metalaxyl-M $4 \%+$ mancozeb $64 \% \mathrm{WP}$. All the isolates were found sensitive to commonly used fungicides but, degree of sensitivity varied significantly. None of the isolate was found resistant to any of the three fungicides i.e. mancozeb $75 \mathrm{WP}$, copper-oxychloride 50WP and metalaxyl-M 4\%+ mancozeb 64\%WP in Himachal Pradesh.

\section{Introduction}

Tomato (Solanum lycopersicum L.) is one of the most important vegetable crops grown widely all over the world. Tomato is the world's one of largest grown vegetable crop. In the world, the area under tomato is 4783 thousand hectares with production of 177042 thousand metric tonnes (Anonymous, 2016). In India, Tomato is grown over an area of 809 thousand hectares with annual production of
19697 thousand MT. In Himachal Pradesh, it is grown over an area of 18.18 thousand hectares with annual production of 415.41 thousand MT (Anonymous, 2017). Tomato is prone to a number of fungal, bacterial and viral diseases. Among these diseases buckeye rot caused by fungal pathogen Phytophthora nicotianae var. parasitica (dastur) Waterhouse is the serious menance to the cultivation of tomato in Himachal Pradesh. In India, this disease has been reported for the first time 
from Solan area of Himachal Pradesh (Jain et al., 1961). Yield loss up to 40 per cent in tomato fruit has been reported due to buckeye rot in Kashmir (Dar et al., 2004). It causes 3040 per cent crop loss under favorable weather conditions (Gupta et al., 2005). Buckeye rot incidence may go upto 90 per cent under high humidity and good rainfall (Gupta and Thind 2006). Buckeye rot disease is mainly managed by the chemicals. Some of the fungicides i.e. mancozeb 75WP (Indofil M-45), copperoxychloride 50WP (Blitox-50) and metalaxylM 4\%+ mancozeb 64\%WP (Ridomil Gold) are widely used for the management of this disease in Himachal Pradesh. However, it is observed that these fungicides were not found equally effective against the disease at different locations, which may be due variation in the pathogen that may lead to loss of sensitivity of the pathogen against these fungicides. It is evident that the fungicides are effective in suppression of buckeye rot, however, no information is available about sensitivity of the pathogen to these fungicides from the state.

The objective of the present study was to find out the variable response of pathogen to these commonly used fungicides in the isolates of Phytophthora nicotianae var. parasitica from Himachal Pradesh. Information regarding sensitivity of pathogen to fungicides will facilitate monitoring potential fungicide resistance development and guide development of more effective programs for managing buckeye rot of tomato by applications of alternate fungicides with different modes of action (Qu et al., 2016).

\section{Materials and Methods}

Collection, Isolation, purification and maintenance of pathogen

Different tomato growing districts i.e. Kullu, Mandi, Kangra, Bilaspur, Solan and Hamirpur of Himachal Pradesh were visited during cropping season 2016-17 for the collection of tomato fruits infected by Phytophthora nicotianae var. parasitica. The fruits showing the symptoms of buckeye rot were collected, placed in paper bags and brought to laboratory for isolation purpose. Pathogen was isolated from naturally infected green fruits of tomato and maintained on potato dextrose agar (PDA) medium. For proper growth of fungal isolate, the culture room temperature was maintained at $25 \pm 1^{\circ} \mathrm{C}$. Each isolate was purified by using single spore method. Pure cultures of each isolate was multiplied on potato dextrose agar medium and used for further studies. Stock cultures were stored at $10^{\circ} \mathrm{C}$ and maintained by regular sub culturing at the interval of 15 days.

\section{In vitro assessment of sensitivity}

Seventeen isolates of $P$. nicotianae var. parasitica were evaluated for their sensitivity to commonly used fungicides i.e. copperoxychloride 50WP (Blitox-50), metalaxyl-M 4\%+ mancozeb 64\%WP (Ridomil Gold) and mancozeb 75WP (Indofil M-45) under in vitro.

These fungicides were evaluated through Poisoned Food Technique (Falck 1907) at concentrations 50, 100, 250, 500 and 750 $\mu \mathrm{g} / \mathrm{ml}$ against all the isolates of pathogen. Double strength PDA medium was prepared in distilled water and sterilized in autoclave at $1.05 \mathrm{~kg} / \mathrm{cm}^{3}$ pressure and $121^{\circ} \mathrm{C}$ for 20 minutes. Simultaneously, double concentration of fungicide was also prepared in sterilized distilled water. Fungicide suspension were added separately to equal quantities of double strength PDA medium aseptically before pouring into Petri plates. These plates were then inoculated with a 5 $\mathrm{mm}$ diameter mycelial bit of the each isolate of pathogen taken from the margin of an actively growing colony and placed in the 
centre of media plates. A control treatment was also maintained in which only plain sterilized distilled water was added to double strength medium. Each treatment was replicated thrice. The inoculated plates were incubated at $25 \pm 1^{\circ} \mathrm{C}$. Regular observations were made and finally colony diameter was measured after 7 days when the control plates were completely covered by the growth of pathogen and per cent mycelial inhibition was determined by formula given by McKinney (1923). On the basis of sensitivity of the pathogen towards these fungicides, seventeen isolates were categorised into three classes i.e. highly sensitive, moderately sensitive and less sensitive. The average mycelial growth of the each isolate was calculated. An isolate was designated as highly sensitive $(\mathrm{H})$, when the isolate exhibited average mycelial growth ranging between 0-20.0 $\mathrm{mm}$.

Moderately sensitive isolate (M) exhibited average mycelial growth ranging between 20.1-40.0 $\mathrm{mm}$ and less sensitive isolate (L) exhibited average mycelial growth ranging between $40.1 \mathrm{~mm}$ and above.

\section{Results and Discussion}

\section{Collection and isolation of pathogen}

More than 25 samples of buckeye rot were collected from six districts (Kullu, Mandi, Kangra, Bilaspur, Solan and Hamirpur) of Himachal Pradesh during 2016-17. From these sample 17 isolates of the pathogen was isolated and purified by using single spore technique. The isolates from different geographical locations were designated as "Pn" and presented in table 1.

\section{Sensitivity to mancozeb 75WP (Indofil M- 45)}

Sensitivity of $P$. nicotianae var. parasitica to mancozeb 75WP (Indofil M-45), the fungicide was tested at different concentrations (50, 100, $250,500 \& 750 \mu \mathrm{g} / \mathrm{ml}$ ) under in vitro and the data on mycelial growth were recorded after 7 days of inoculation (Table 2). The data revealed that there was significant difference among isolates in sensitivity to mancozeb $75 \mathrm{WP}$ at different doses. At $50 \mu \mathrm{g} / \mathrm{ml}$, isolate Pn-13 showed highest mycelial growth i.e. $73.00 \mathrm{~mm}$, followed by isolate Pn-8 which showed $65.17 \mathrm{~mm}$ mycelial growth. Isolate Pn-3 showed lowest (17.00 $\mathrm{mm})$ mycelial growth at $50 \mu \mathrm{g} / \mathrm{ml}$ while, other isolates showed mycelial growth ranging from 21.00$60.00 \mathrm{~mm}$. At $100 \mu \mathrm{g} / \mathrm{ml}$, isolate $\mathrm{Pn}-13$ showed highest mycelial growth i.e. $51.50 \mathrm{~mm}$ followed by Pn-8 which showed $50.50 \mathrm{~mm}$ mycelial growth, however, Pn-3 showed lowest $(13.17 \mathrm{~mm})$ mycelial growth. Other isolates at $100 \mu \mathrm{g} / \mathrm{ml}$ showed mycelial growth ranging between 16.17 to $45.67 \mathrm{~mm}$. Isolate Pn-17 was completely inhibited at $250 \mu \mathrm{g} / \mathrm{ml}$ whereas, remaining isolates showed mycelial growth ranging from 8.50 to $41.33 \mathrm{~mm}$. At $500 \mu \mathrm{g} / \mathrm{ml}$, isolate Pn-3, Pn-4, Pn-5, Pn-7, Pn9, Pn-10, Pn-12 and Pn-14 showed complete inhibition of mycelial growth however, in other isolates mycelial growth ranged from $7.33-18.00 \mathrm{~mm}$. At $750 \mu \mathrm{g} / \mathrm{ml}$, no mycelial growth was observed in any of the isolates.

Seventeen isolates of $P$. nicotianae var. parasitica were categorized into three groups of sensitivity i.e. highly, moderately and less sensitive. (Table 3) Out of these three sensitivity groups, PnG1 consisted nine isolates (Pn-3, Pn-4, Pn-5, Pn-7, Pn-9, Pn-10, Pn-12, Pn-14 and Pn-17), which were observed highly sensitive to mancozeb 75WP. The average mycelial growth of isolates of this group ranged between 0-20.0 per cent. In the second group $(\mathrm{PnG} 2)$ seven isolates $(\mathrm{Pn}-1$, Pn-2, Pn-6, Pn-8, Pn-11, Pn-15 and Pn-16) were placed, which were moderately sensitive to mancozeb $75 \mathrm{WP}$. The average mycelial growth of isolates of this group ranged between 20.1-40.0 per cent. In group PnG3, 
the isolates having average mycelial growth 40.1 per cent or above were placed and this group is having only one isolate Pn-13.

\section{Sensitivity to copper-oxychloride 50WP (Blitox-50)}

Sensitivity of $P$. nicotianae var. parasitica to copper-oxychloride 50WP (Blitox-50), the fungicide was tested at different concentrations $(50,100,250,500$ and 750 $\mu \mathrm{g} / \mathrm{ml}$ ) under in vitro and the data on mycelial growth were recorded after 7 days of inoculation and presented in table 4 . The data revealed that at $50 \mu \mathrm{g} / \mathrm{ml}$, isolate Pn-6 showed highest mycelial growth i.e. $67.67 \mathrm{~mm}$ followed by $\mathrm{Pn}-8$ which gave $64.50 \mathrm{~mm}$ mycelial growth. Isolate Pn-9 provided lowest $(23.33 \mathrm{~mm})$ mycelial growth at $50 \mu \mathrm{g} / \mathrm{ml}$ while, other isolates showed mycelial growth ranging between 25.17 to $62.33 \mathrm{~mm}$. At 100 $\mu \mathrm{g} / \mathrm{ml}$, isolate $\mathrm{Pn}-8$ gave highest $(61.17 \mathrm{~mm})$ mycelial growth followed by Pn-6 which provided $50.33 \mathrm{~mm}$ mycelial growth. Isolate Pn-9 provided lowest mycelial growth i.e. $13.67 \mathrm{~mm}$, however other isolates showed mycelial growth ranging from 21.00-43.50 $\mathrm{mm}$ at $100 \mu \mathrm{g} / \mathrm{ml}$. Isolate Pn-2, Pn-3, Pn-9, Pn-10, Pn-11, Pn-13 and Pn-16 provided complete inhibition of mycelial growth at 250 $\mu \mathrm{g} / \mathrm{ml}$. Other isolates at $250 \mu \mathrm{g} / \mathrm{ml}$ showed mycelial growth ranging from 8.17- 40.67 $\mathrm{mm}$. At $500 \mu \mathrm{g} / \mathrm{ml}$, isolate Pn-1, Pn-5, Pn-7, Pn-8, Pn-12, Pn-14, Pn-15 and Pn-17 also showed complete inhibition of mycelial growth followed by Pn-4 and Pn-6 with 19.17 and $18.85 \mathrm{~mm}$ mycelial growth. At $750 \mu \mathrm{g} / \mathrm{ml}$, no mycelial growth was observed in any of the isolates. Seventeen isolates of $P$. nicotianae var. parasitica were categorized into three groups of sensitivity i.e. highly, moderately and less sensitive (Table 5). Out of these three sensitivity groups, PnG1 consisted seven isolates (Pn-2, Pn-3, Pn-9, Pn-10, Pn-11, Pn13 and Pn-16), which were observed highly sensitive to copper-oxychloride 50WP. The average mycelial growth of isolates of this group ranged between 0-20 per cent. In second group (PnG2) nine isolates (Pn-1, Pn-4, Pn-5, Pn-7, Pn-8, Pn-12, Pn-14, Pn-15 and Pn-17) were placed which were moderately sensitive to copper-oxychloride 50WP. The average mycelial growth of isolates of this group ranged between 20.1-40.0 per cent. In group PnG3, only one isolate Pn-6 was placed because the average mycelial growth was more than 40.1 per cent.

\section{Sensitivity to metalaxyl-M 4\%+ mancozeb 64\% WP (Ridomil Gold)}

Sensitivity of $P$. nicotianae var. parasitica to metalaxyl-M 4\%+ mancozeb $64 \% \mathrm{WP}$ (Ridomil Gold), the fungicide was tested at different concentrations $(50,100,250,500 \&$ $750 \mu \mathrm{g} / \mathrm{ml}$ ) under in vitro and the data on mycelial growth was recorded after 7 days of inoculation and presented in table 6. The data revealed that at $50 \mu \mathrm{g} / \mathrm{ml}$, isolate Pn1 showed highest mycelial growth i.e. $59.50 \mathrm{~mm}$, followed by $\mathrm{Pn}-8$ which provided $58.50 \mathrm{~mm}$ mycelial growth. Isolate Pn-5 gave lowest $(16.67 \mathrm{~mm})$ mycelial growth at $50 \mu \mathrm{g} / \mathrm{ml}$ while, other isolates showed mycelial growth ranging from $16.83-52.50 \mathrm{~mm}$. At $100 \mu \mathrm{g} / \mathrm{ml}$, isolate Pn-17 gave complete inhibition of mycelial growth. Minimum mycelial growth was given by $\mathrm{Pn}-2(6.67 \mathrm{~mm})$ while, other isolates provided mycelial growth ranging from $10.67-51.83 \mathrm{~mm}$ at $100 \mu \mathrm{g} / \mathrm{ml}$. Isolate Pn-2, Pn-3, Pn-4, Pn-5, Pn-6, Pn-10, Pn-12, Pn-13 and Pn-15 completely inhibited the mycelial growth at $250 \mu \mathrm{g} / \mathrm{ml}$. Isolate Pn-11 gave minimum mycelial growth i.e. $10.17 \mathrm{~mm}$ at $250 \mu \mathrm{g} / \mathrm{ml}$ while, other isolates showed mycelial growth ranging from 13.67-40.24 $\mathrm{mm}$ at $250 \mu \mathrm{g} / \mathrm{ml}$. At $500 \mu \mathrm{g} / \mathrm{ml}$, isolate Pn-1, Pn-7, Pn-9, Pn-11, Pn-14 and Pn-16 gave complete inhibition of mycelial growth and only one isolate Pn-8 showed $9.50 \mathrm{~mm}$ mycelial growth. At $750 \mu \mathrm{g} / \mathrm{ml}$, all the isolates completely inhibited the mycelial growth. 
Table.1 Phytophthora nicotianae var. parasitica isolates from different locations of Himachal Pradesh

\begin{tabular}{|c|c|c|c|}
\hline District & S. No & Isolate Designation & Location \\
\hline \multirow[t]{3}{*}{ Mandi } & 1 & $\mathrm{Pn}-1$ & Jhiri \\
\hline & 2 & $\mathrm{Pn}-3$ & Nagwain \\
\hline & 3 & Pn-14 & Takoli \\
\hline \multirow[t]{6}{*}{ Kangra } & 4 & Pn-13 & Jamanabad \\
\hline & 5 & Pn-2 & Tanda \\
\hline & 6 & Pn-9 & Harzana \\
\hline & 7 & Pn-10 & Kohli \\
\hline & 8 & Pn-11 & Nagrota \\
\hline & 9 & $\mathrm{Pn}-4$ & Palampur \\
\hline Bilaspur & 10 & Pn-5 & Namhol \\
\hline Kullu & 11 & Pn-6 & Bajaura \\
\hline \multirow[t]{5}{*}{ Solan } & 12 & $\mathrm{Pn}-7$ & Badlag \\
\hline & 13 & $\mathrm{Pn}-8$ & Ochghat \\
\hline & 14 & Pn-15 & Dharmpur \\
\hline & 15 & Pn-16 & Saproon \\
\hline & 26 & Pn-17 & Lohanji \\
\hline Hamirpur & 17 & Pn-12 & Bara \\
\hline
\end{tabular}


Table.2 Sensitivity of Phytophthora nicotianae var. parasitica isolates to mancozeb 75WP (Indofil M-45)

\begin{tabular}{|c|c|c|c|c|c|c|c|}
\hline \multirow[t]{2}{*}{ Isolates } & \multicolumn{6}{|c|}{ Mycelial growth $(\mathrm{mm})$ at different concentrations $(\mu \mathrm{g} / \mathrm{ml})$} & \multirow{2}{*}{$\begin{array}{c}\text { Mancozeb } \\
75 W P \\
\text { sensitivity }\end{array}$} \\
\hline & 50 & 100 & 250 & 500 & $750 *$ & Average & \\
\hline Pn-1 & 50.67 & 44.00 & $\begin{array}{l}29.33 \\
(5.50)\end{array}$ & $\begin{array}{l}16.33 \\
(4.16)\end{array}$ & $\begin{array}{c}0.00 \\
(1.00)\end{array}$ & 35.08 & $\mathrm{M}$ \\
\hline Pn-2 & 40.17 & 36.00 & $\begin{array}{l}28.67 \\
(5.44)\end{array}$ & $\begin{array}{c}8.83 \\
(3.13)\end{array}$ & $\begin{array}{c}0.00 \\
(1.00)\end{array}$ & 28.42 & M \\
\hline Pn-3 & 17.00 & 13.17 & $\begin{array}{c}8.50 \\
(3.08)\end{array}$ & $\begin{array}{c}0.00 \\
(1.00)\end{array}$ & $\begin{array}{c}0.00 \\
(1.00)\end{array}$ & 9.67 & $\mathrm{H}$ \\
\hline Pn-4 & 26.83 & 22.17 & $\begin{array}{l}19.17 \\
(4.48)\end{array}$ & $\begin{array}{c}0.00 \\
(1.00)\end{array}$ & $\begin{array}{c}0.00 \\
(1.00)\end{array}$ & 17.04 & $\mathrm{H}$ \\
\hline Pn-5 & 31.33 & 22.00 & $\begin{array}{l}16.00 \\
(4.12)\end{array}$ & $\begin{array}{c}0.00 \\
(1.00)\end{array}$ & $\begin{array}{c}0.00 \\
(1.00)\end{array}$ & 17.33 & $\mathrm{H}$ \\
\hline Pn-6 & 40.83 & 34.50 & $\begin{array}{l}27.17 \\
(5.30)\end{array}$ & $\begin{array}{l}10.00 \\
(3.31)\end{array}$ & $\begin{array}{c}0.00 \\
(1.00)\end{array}$ & 28.13 & $\mathrm{M}$ \\
\hline Pn-7 & 24.50 & 20.33 & $\begin{array}{l}11.00 \\
(4.06)\end{array}$ & $\begin{array}{c}0.00 \\
(1.00)\end{array}$ & $\begin{array}{c}0.00 \\
(1.00)\end{array}$ & 15.08 & $\mathrm{H}$ \\
\hline Pn-8 & 65.17 & 50.50 & $\begin{array}{l}27.17 \\
(5.30)\end{array}$ & $\begin{array}{l}13.17 \\
(3.76)\end{array}$ & $\begin{array}{c}0.00 \\
(1.00)\end{array}$ & 39.00 & M \\
\hline Pn-9 & 25.67 & 21.00 & $\begin{array}{l}11.00 \\
(3.46)\end{array}$ & $\begin{array}{c}0.00 \\
(1.00)\end{array}$ & $\begin{array}{c}0.00 \\
(1.00)\end{array}$ & 14.42 & $\mathrm{H}$ \\
\hline Pn-10 & 21.00 & 16.17 & $\begin{array}{l}12.50 \\
(3.67)\end{array}$ & $\begin{array}{c}0.00 \\
(1.00)\end{array}$ & $\begin{array}{c}0.00 \\
(1.00)\end{array}$ & 12.42 & $\mathrm{H}$ \\
\hline Pn-11 & 41.17 & 30.83 & $\begin{array}{l}22.00 \\
(4.79)\end{array}$ & $\begin{array}{c}8.57 \\
(3.09)\end{array}$ & $\begin{array}{c}0.00 \\
(1.00)\end{array}$ & 25.64 & $\mathrm{M}$ \\
\hline Pn-12 & 33.00 & 27.50 & $\begin{array}{r}19.00 \\
(4.47)\end{array}$ & $\begin{array}{c}0.00 \\
(1.00)\end{array}$ & $\begin{array}{c}0.00 \\
(1.00)\end{array}$ & 19.88 & $\mathrm{H}$ \\
\hline Pn-13 & 73.00 & 51.50 & $\begin{array}{l}41.33 \\
(6.50)\end{array}$ & $\begin{array}{l}18.00 \\
(4.35)\end{array}$ & $\begin{array}{c}0.00 \\
(1.00)\end{array}$ & 45.96 & $\mathrm{~L}$ \\
\hline Pn-14 & 31.67 & 22.67 & $\begin{array}{r}14.33 \\
(3.91)\end{array}$ & $\begin{array}{c}0.00 \\
(1.00)\end{array}$ & $\begin{array}{c}0.00 \\
(1.00)\end{array}$ & 17.17 & $\mathrm{H}$ \\
\hline Pn-15 & 60.00 & 45.67 & $\begin{array}{l}30.67 \\
(5.62)\end{array}$ & $\begin{array}{l}15.00 \\
(3.99)\end{array}$ & $\begin{array}{c}0.00 \\
(1.00)\end{array}$ & 37.84 & $\mathrm{M}$ \\
\hline Pn-16 & 56.50 & 41.50 & $\begin{array}{l}20.83 \\
(4.67)\end{array}$ & $\begin{array}{c}7.33 \\
(2.88)\end{array}$ & $\begin{array}{c}0.00 \\
(1.00)\end{array}$ & 31.54 & M \\
\hline Pn-17 & 39.17 & 20.67 & $\begin{array}{c}0.00 \\
(1.00)\end{array}$ & $\begin{array}{c}0.00 \\
(1.00)\end{array}$ & $\begin{array}{c}0.00 \\
(1.00)\end{array}$ & 14.96 & $\mathrm{H}$ \\
\hline Control & 90 & 90 & $\begin{array}{c}90 \\
(9.53)\end{array}$ & $\begin{array}{c}90 \\
(9.53)\end{array}$ & $\begin{array}{c}90 \\
(9.53)\end{array}$ & - & - \\
\hline $\operatorname{CD}(p=0.05)$ & 1.82 & 1.44 & 0.16 & 0.14 & 0.00 & & \\
\hline
\end{tabular}


Table.3 Grouping of Phytophthora nicotianae var. parasitica isolates on the basis of sensitivity to mancozeb 75WP (Indofil M-45)

\begin{tabular}{|l|l|l|}
\hline Group no. & Isolates & Sensitivity to Mancozeb 75WP \\
\hline PnG1 & $\begin{array}{l}\mathrm{Pn}-3, \mathrm{Pn}-4, \mathrm{Pn}-5, \mathrm{Pn}-7, \mathrm{Pn}-9, \mathrm{Pn}-10, \mathrm{Pn}-12, \mathrm{Pn}- \\
14 \text { and } \mathrm{Pn}-17\end{array}$ & Highly sensitive \\
\hline PnG2 & $\mathrm{Pn}-1, \mathrm{Pn}-2, \mathrm{Pn}-6, \mathrm{Pn}-8, \mathrm{Pn}-11, \mathrm{Pn}-15$ and Pn-16 & Moderately sensitive \\
\hline PnG3 & $\mathrm{Pn}-13$ & Less sensitive \\
\hline
\end{tabular}

Table.4 Sensitivity of Phytophthora nicotianae var. parasitica isolates to copper-oxychloride 50WP (Blitox-50)

\begin{tabular}{|c|c|c|c|c|c|c|c|}
\hline \multirow[t]{2}{*}{ Isolates } & \multicolumn{6}{|c|}{ Mycelial growth $(\mathrm{mm})$ at different concentrations $(\mu \mathrm{g} / \mathrm{ml})$} & \multirow{2}{*}{$\begin{array}{l}\text { Copper-oxychloride } \\
50 W P \text { sensitivity }\end{array}$} \\
\hline & 50 & 100 & 250 & 500 & $750 *$ & Average & \\
\hline Pn-1 & 49.00 & 43.50 & $\begin{array}{c}8.17 \\
(3.02)\end{array}$ & $\begin{array}{c}0.00 \\
(1.00)\end{array}$ & $\begin{array}{c}0.00 \\
(1.00)\end{array}$ & 25.17 & M \\
\hline Pn-2 & 32.00 & 22.83 & $\begin{array}{c}0.00 \\
(1.00)\end{array}$ & $\begin{array}{c}0.00 \\
(1.00)\end{array}$ & $\begin{array}{c}0.00 \\
(1.00)\end{array}$ & 13.71 & $\mathrm{H}$ \\
\hline Pn-3 & 25.50 & 21.50 & $\begin{array}{c}0.00 \\
(1.00)\end{array}$ & $\begin{array}{c}0.00 \\
(1.00)\end{array}$ & $\begin{array}{c}0.00 \\
(1.00)\end{array}$ & 11.75 & $\mathrm{H}$ \\
\hline Pn-4 & 43.00 & 37.00 & $\begin{array}{l}24.00 \\
(4.99)\end{array}$ & $\begin{array}{l}19.17 \\
(4.49)\end{array}$ & $\begin{array}{c}0.00 \\
(1.00)\end{array}$ & 30.79 & M \\
\hline Pn-5 & 51.67 & 41.33 & $\begin{array}{l}14.00 \\
(3.87)\end{array}$ & $\begin{array}{c}0.00 \\
(1.00)\end{array}$ & $\begin{array}{c}0.00 \\
(1.00)\end{array}$ & 26.75 & M \\
\hline Pn-6 & 67.67 & 50.33 & $\begin{array}{l}40.67 \\
(6.45)\end{array}$ & $\begin{array}{l}18.85 \\
(4.45)\end{array}$ & $\begin{array}{c}0.00 \\
(1.00)\end{array}$ & 44.38 & $\mathrm{~L}$ \\
\hline Pn-7 & 62.33 & 41.33 & $\begin{array}{l}37.77 \\
(6.22)\end{array}$ & $\begin{array}{c}0.00 \\
(1.00)\end{array}$ & $\begin{array}{c}0.00 \\
(1.00)\end{array}$ & 35.36 & M \\
\hline Pn-8 & 64.50 & 61.17 & $\begin{array}{l}22.83 \\
(4.88)\end{array}$ & $\begin{array}{c}0.00 \\
(1.00)\end{array}$ & $\begin{array}{c}0.00 \\
(1.00)\end{array}$ & 37.13 & M \\
\hline Pn-9 & 23.33 & 13.67 & $\begin{array}{c}0.00 \\
(1.00)\end{array}$ & $\begin{array}{c}0.00 \\
(1.00)\end{array}$ & $\begin{array}{c}0.00 \\
(1.00)\end{array}$ & 9.25 & $\mathrm{H}$ \\
\hline Pn-10 & 32.67 & 25.17 & $\begin{array}{c}0.00 \\
(1.00)\end{array}$ & $\begin{array}{c}0.00 \\
(1.00)\end{array}$ & $\begin{array}{c}0.00 \\
(1.00)\end{array}$ & 14.46 & $\mathrm{H}$ \\
\hline Pn-11 & 34.17 & 27.33 & $\begin{array}{c}0.00 \\
(1.00)\end{array}$ & $\begin{array}{c}0.00 \\
(1.00)\end{array}$ & $\begin{array}{c}0.00 \\
(1.00)\end{array}$ & 15.38 & $\mathrm{H}$ \\
\hline Pn-12 & 50.33 & 41.17 & $\begin{array}{l}20.83 \\
(4.67)\end{array}$ & $\begin{array}{c}0.00 \\
(1.00)\end{array}$ & $\begin{array}{c}0.00 \\
(1.00)\end{array}$ & 28.08 & M \\
\hline Pn-13 & 25.17 & 21.00 & $\begin{array}{c}0.00 \\
(1.00)\end{array}$ & $\begin{array}{c}0.00 \\
(1.00)\end{array}$ & $\begin{array}{c}0.00 \\
(1.00)\end{array}$ & 11.54 & $\mathrm{H}$ \\
\hline Pn-14 & 41.67 & 22.83 & $\begin{array}{l}16.50 \\
(4.18)\end{array}$ & $\begin{array}{c}0.00 \\
(1.00)\end{array}$ & $\begin{array}{c}0.00 \\
(1.00)\end{array}$ & 20.25 & M \\
\hline Pn-15 & 55.00 & 34.50 & $\begin{array}{l}24.00 \\
(5.00)\end{array}$ & $\begin{array}{c}0.00 \\
(1.00)\end{array}$ & $\begin{array}{c}0.00 \\
(1.00)\end{array}$ & 28.38 & M \\
\hline Pn-16 & 31.33 & 23.83 & $\begin{array}{c}0.00 \\
(1.00)\end{array}$ & $\begin{array}{c}0.00 \\
(1.00)\end{array}$ & $\begin{array}{c}0.00 \\
(1.00)\end{array}$ & 13.79 & $\mathrm{H}$ \\
\hline Pn-17 & 58.00 & 30.67 & $\begin{array}{l}15.00 \\
(3.99)\end{array}$ & $\begin{array}{c}0.00 \\
(1.00)\end{array}$ & $\begin{array}{c}0.00 \\
(1.00)\end{array}$ & 25.92 & M \\
\hline Control & 90.00 & 90.00 & $\begin{array}{l}90.00 \\
(9.53)\end{array}$ & $\begin{array}{l}90.00 \\
(9.53)\end{array}$ & $\begin{array}{l}90.00 \\
(9.53)\end{array}$ & - & - \\
\hline $\begin{array}{l}\text { CD } \\
(p=0.05)\end{array}$ & 1.63 & 1.56 & 0.12 & 0.04 & 0.00 & & \\
\hline
\end{tabular}


Table.5 Grouping of Phytophthora nicotianae var. parasitica isolates on the basis of sensitivity to copper-oxychloride 50WP (Blitox-50)

\begin{tabular}{|l|c|c|}
\hline Group no. & Isolates & $\begin{array}{c}\text { Sensitivity to Copper-oxychloride } \\
\text { 50WP }\end{array}$ \\
\hline PnG1 & $\begin{array}{c}\text { Pn-2, Pn-3, Pn- 9, Pn-10, Pn-11, Pn-13 } \\
\text { and Pn-16 }\end{array}$ & Highly sensitive \\
\hline PnG2 & $\begin{array}{c}\text { Pn-1, Pn-4, Pn-5, Pn-7, Pn-8, Pn-12, } \\
\text { Pn-14, Pn-15 and Pn-17 }\end{array}$ & Moderately sensitive \\
\hline PnG3 & Pn- 6 & Less sensitive \\
\hline
\end{tabular}

Table.6 Sensitivity of Phytophthora nicotianae var. parasitica isolates to metalaxyl-M 4\%+ mancozeb 64\%WP (Ridomil Gold)

\begin{tabular}{|c|c|c|c|c|c|c|c|}
\hline \multirow[t]{2}{*}{ Isolates } & \multicolumn{6}{|c|}{ Mycelial growth $(\mathrm{mm})$ at different concentrations $(\mu \mathrm{g} / \mathrm{ml})$} & \multirow{2}{*}{$\begin{array}{c}\text { Metalaxyl-M 4\%+mancozeb } \\
64 \% \text { WP Sensitivity }\end{array}$} \\
\hline & 50 & 100 & 250 & 500 & $750 *$ & Average & \\
\hline Pn-1 & 59.50 & $\begin{array}{l}34.50 \\
(5.95)\end{array}$ & $\begin{array}{l}19.50 \\
(4.52)\end{array}$ & $\begin{array}{c}0.00 \\
(1.00)\end{array}$ & $\begin{array}{c}0.00 \\
(1.00)\end{array}$ & 28.38 & M \\
\hline Pn-2 & 21.00 & $\begin{array}{c}6.67 \\
(2.76)\end{array}$ & $\begin{array}{c}0.00 \\
(1.00)\end{array}$ & $\begin{array}{c}0.00 \\
(1.00)\end{array}$ & $\begin{array}{c}0.00 \\
(1.00)\end{array}$ & 6.92 & $\mathrm{H}$ \\
\hline Pn-3 & 21.33 & $\begin{array}{l}16.33 \\
(4.16)\end{array}$ & $\begin{array}{c}0.00 \\
(1.00)\end{array}$ & $\begin{array}{c}0.00 \\
(1.00)\end{array}$ & $\begin{array}{c}0.00 \\
(1.00)\end{array}$ & 9.42 & $\mathrm{H}$ \\
\hline Pn-4 & 34.50 & $\begin{array}{l}16.67 \\
(4.20)\end{array}$ & $\begin{array}{c}0.00 \\
(1.00)\end{array}$ & $\begin{array}{c}0.00 \\
(1.00)\end{array}$ & $\begin{array}{c}0.00 \\
(1.00)\end{array}$ & 12.79 & $\mathrm{H}$ \\
\hline Pn-5 & 16.67 & $\begin{array}{l}12.50 \\
(3.67)\end{array}$ & $\begin{array}{c}0.00 \\
(1.00)\end{array}$ & $\begin{array}{c}0.00 \\
(1.00)\end{array}$ & $\begin{array}{c}0.00 \\
(1.00)\end{array}$ & 7.29 & $\mathrm{H}$ \\
\hline Pn-6 & 22.83 & $\begin{array}{l}18.00 \\
(4.35)\end{array}$ & $\begin{array}{c}0.00 \\
(1.00)\end{array}$ & $\begin{array}{c}0.00 \\
(1.00)\end{array}$ & $\begin{array}{c}0.00 \\
(1.00)\end{array}$ & 10.21 & $\mathrm{H}$ \\
\hline Pn-7 & 50.00 & $\begin{array}{l}31.67 \\
(5.71)\end{array}$ & $\begin{array}{l}14.22 \\
(3.90)\end{array}$ & $\begin{array}{c}0.00 \\
(1.00)\end{array}$ & $\begin{array}{c}0.00 \\
(1.00)\end{array}$ & 23.97 & M \\
\hline Pn-8 & 58.50 & $\begin{array}{l}51.83 \\
(7.26)\end{array}$ & $\begin{array}{l}40.24 \\
(6.42)\end{array}$ & $\begin{array}{c}9.50 \\
(3.23)\end{array}$ & $\begin{array}{c}0.00 \\
(1.00)\end{array}$ & 40.02 & $\mathrm{~L}$ \\
\hline Pn-9 & 41.67 & $\begin{array}{l}30.67 \\
(5.62)\end{array}$ & $\begin{array}{l}13.67 \\
(3.82)\end{array}$ & $\begin{array}{c}0.00 \\
(1.00)\end{array}$ & $\begin{array}{c}0.00 \\
(1.00)\end{array}$ & 21.50 & M \\
\hline Pn-10 & 31.00 & $\begin{array}{l}13.67 \\
(3.82) \\
\end{array}$ & $\begin{array}{c}0.00 \\
(1.00)\end{array}$ & $\begin{array}{c}0.00 \\
(1.00)\end{array}$ & $\begin{array}{c}0.00 \\
(1.00)\end{array}$ & 11.17 & $\mathrm{H}$ \\
\hline Pn-11 & 22.83 & $\begin{array}{l}16.00 \\
(4.12)\end{array}$ & $\begin{array}{l}10.17 \\
(3.33)\end{array}$ & $\begin{array}{c}0.00 \\
(1.00)\end{array}$ & $\begin{array}{c}0.00 \\
(1.00)\end{array}$ & 12.25 & $\mathrm{H}$ \\
\hline Pn-12 & 20.67 & $\begin{array}{l}15.33 \\
(4.04)\end{array}$ & $\begin{array}{c}0.00 \\
(1.00)\end{array}$ & $\begin{array}{c}0.00 \\
(1.00)\end{array}$ & $\begin{array}{c}0.00 \\
(1.00)\end{array}$ & 9.00 & $\mathrm{H}$ \\
\hline Pn-13 & 16.83 & $\begin{array}{l}10.67 \\
(3.41)\end{array}$ & $\begin{array}{c}0.00 \\
(1.00)\end{array}$ & $\begin{array}{c}0.00 \\
(1.00)\end{array}$ & $\begin{array}{c}0.00 \\
(1.00)\end{array}$ & 6.88 & $\mathrm{H}$ \\
\hline Pn-14 & 52.50 & $\begin{array}{l}25.50 \\
(5.14)\end{array}$ & $\begin{array}{l}18.17 \\
(4.37)\end{array}$ & $\begin{array}{c}0.00 \\
(1.00)\end{array}$ & $\begin{array}{c}0.00 \\
(1.00)\end{array}$ & 24.04 & M \\
\hline Pn-15 & 20.33 & $\begin{array}{l}14.00 \\
(3.87)\end{array}$ & $\begin{array}{c}0.00 \\
(1.00)\end{array}$ & $\begin{array}{c}0.00 \\
(1.00)\end{array}$ & $\begin{array}{c}0.00 \\
(1.00)\end{array}$ & 8.58 & $\mathrm{H}$ \\
\hline Pn-16 & 47.00 & $\begin{array}{l}30.67 \\
(5.62)\end{array}$ & $\begin{array}{l}21.15 \\
(4.70)\end{array}$ & $\begin{array}{c}0.00 \\
(1.00)\end{array}$ & $\begin{array}{c}0.00 \\
(1.00)\end{array}$ & 24.70 & M \\
\hline Pn-17 & 21.67 & $\begin{array}{c}0.00 \\
(1.00)\end{array}$ & $\begin{array}{c}0.00 \\
(1.00)\end{array}$ & $\begin{array}{c}0.00 \\
(1.00)\end{array}$ & $\begin{array}{c}0.00 \\
(1.00)\end{array}$ & 5.42 & $\mathrm{H}$ \\
\hline Control & 90.00 & $\begin{array}{l}90.00 \\
(9.53)\end{array}$ & $\begin{array}{l}90.00 \\
(9.53)\end{array}$ & $\begin{array}{l}90.00 \\
(9.53)\end{array}$ & $\begin{array}{l}90.00 \\
(9.53)\end{array}$ & - & - \\
\hline $\begin{array}{l}\text { CD } \\
(p=0.05)\end{array}$ & 1.28 & 0.14 & 0.10 & 0.06 & 0.00 & & \\
\hline
\end{tabular}

$\mathrm{L}=$ less sensitive, $\mathrm{M}=$ moderately sensitive, $\mathrm{H}=$ highly sensitive

* not included for calculating average 
Table.7 Grouping of Phytophthora nicotianae var. parasitica isolates on the basis of sensitivity to metalaxyl-M 4\%+ mancozeb 64\%WP (Ridomil Gold)

\begin{tabular}{|l|l|c|}
\hline Group no. & Isolates & $\begin{array}{c}\text { Sensitivity to metalaxyl-M } \\
\mathbf{4 \%}+\text { mancozeb 64\% WP }\end{array}$ \\
\hline PnG1 & $\begin{array}{l}\text { Pn-2, Pn-3, Pn-4, Pn-5, Pn-6, Pn-10, Pn- } \\
11, \mathrm{Pn}-12, \mathrm{Pn}-13, \mathrm{Pn}-15 \text { and Pn-17 }\end{array}$ & Highly sensitive \\
\hline PnG2 & Pn-1, Pn-7, Pn-9, Pn-14 and Pn-16 & Moderately sensitive \\
\hline PnG3 & Pn- 8 & Less sensitive \\
\hline
\end{tabular}

Seventeen isolates of $P$. nicotianae var. parasitica were categorized into three groups of sensitivity i.e. highly, moderately and less sensitive (Table 7). Group PnG1 accommodated eleven isolates (Pn-2, Pn-3, Pn-4, Pn-5, Pn-6, Pn-10, Pn-11, Pn-12, Pn-13, Pn-15 and Pn-17), which were highly sensitive to metalaxyl-M $4 \%+$ mancozeb 64\%WP. The average mycelial growth of isolates of this group ranged between 0-20.0 per cent. Five isolates (Pn-1, Pn-7, Pn-9, Pn-14 and Pn-16), which were moderately sensitive to metalaxyl-M 4\%+ mancozeb $64 \% \mathrm{WP}$ were placed in group PnG2. The average mycelial growth of isolates of this group ranged between 20.1-40.0 per cent. In group PnG3, only one isolate Pn-8 was placed having average mycelial growth more than 40.1 per cent.

From the above data it was concluded that all the isolates were sensitive to commonly used fungicides but, degree of sensitivity varied significantly. Out of seventeen isolates, nine isolates (Pn -3, Pn-4, Pn-5, Pn-7, Pn- 9, Pn-10, Pn-12, Pn-14 and Pn-17) were highly sensitive, seven isolates (Pn-1, Pn-2, Pn-6, Pn-8, Pn-11, Pn-15 and Pn-16) were moderately sensitive and one isolate Pn-13 was less sensitive. Out of seventeen isolates, seven isolates ( $\mathrm{Pn}-2, \mathrm{Pn}-3$, Pn- 9, Pn-10, Pn-11, Pn-13 and Pn-16) were highly sensitive, nine isolates (Pn-1, Pn-4, Pn-5, Pn-7, Pn-8, Pn-12, Pn-14, Pn-15 and Pn-17) were moderately sensitive and one isolate Pn-6 was less sensitive. Out of seventeen isolates, eleven isolates (Pn -2, Pn-3, Pn-4, Pn- 5, Pn-6, Pn-10, Pn-11, Pn-12, Pn-13, Pn-15 and Pn-17) were highly sensitive, five isolates (Pn-1, Pn-7, Pn-9, Pn-14 and Pn-16) were moderately sensitive and only one isolate Pn-8 was less sensitive.

However, none of the isolate was found resistant to any of the three fungicides i.e. mancozeb 75WP, copper-oxychloride 50WP and metalaxyl-M 4\%+ mancozeb 64\%WP. The variation in effectiveness of these fungicides at different locations may be due to the differences in the sensitivity among the different isolates of the pathogens.

The variation in sensitivity may be due to the continuous and long term use of these fungicides by the farmers against the pathogen in particular areas. The sensitivity of the Phytophthora spp. against the different fungicides has been reported by different workers. Sujkowaski et al., (1995) reported that the sensitivity of $P$. infestans isolates was lowest for cymoxanil, low for chlorothalonil and higher for metalaxyl. Kato et al., (1997) reported that there were significant differences among isolates of $P$. infestans for degree of sensitivity to one fungicide individually, but there were no significant difference among the isolates for degree of sensitivity to mancozeb and chlorothalonil. Jaarsveld et al., (2002) screened one hundred and thirty-two isolates of $P$. nicotianae for sensitivity to metalaxyl and found that growth of most isolates was inhibited completely at 1.0 a.i $\mu \mathrm{g} / \mathrm{ml}$. Hu et al., (2008) screened 95 isolates of $P$. nicotianae for their sensitivity to mefenoxam at 100 a.i. $\mu \mathrm{g} / \mathrm{ml}$. Twenty- five isolates were highly resistant, four were intermediately resistant and sixty- six isolates were observed sensitive. Wang et al., (2013) studied the mefenoxam sensitivity in the 
$P$. parasitica var. nicotianae and found that 41.9 per cent isolates were intermediately resistant and 58.1 per cent were sensitive to mefenoxam, however, no isolate was found resistant. Qu et al., (2016) studied twenty isolates of $P$. nicotianae from tobacco to determine their sensitivity to fluopicolide, mandipropamid and oxathiapiprolin. Results indicated that $P$. nicotianae isolates were sensitive to the fungicides, with lower $\mathrm{EC}_{50}$ for oxathiapiprolin than for fluopicolide and mandipropamid.

\section{References}

Brent KJ and Hollomon DW. 2007. Fungicide resistance: The assessment of risk. FRAC Monograph 2 (revised). (At: http://www.frac.info/frac/index.htm) [note: also "detection and verification"]

Dar GM, Dar GH, Baba RA and Munshi NA. 2004. Causes and management of tomato fruit rots in Kashmir. Applied Biological Research 6: 22-26

Falck R. 1907. Wachstumgesetze, Wachstumfaktoren und Temperaturewerte des Holzzerstoerenden Mycelium 1: 53-154

Gupta SK and Thind TS. 2006. Disease problems in vegetable production. Kalyani Publishers, New Delhi. Indian Phytopathology 342

Gupta SK, Upmanyu S and Sharma RC. 2005. Biology, epidemiology and management of buckeye rot of tomato. In: challenging problems in horticultural and forest pathology. Sharma RC and Sharma JN (eds.). Indus Publishing Co., New Delhi. Indian Phytopathology 183-199

$\mathrm{Hu} \mathrm{JH}$, Hong CX, Stromberg EL and Moorman GW. 2008. Mefenoxam sensitivity and fitness analysis of Phytophthora nicotianae isolates from nurseries in Virginia, USA. Plant Pathology 57: 728736

Jaarsveld EJV, Wingfield MJ and Drenth A. 2002. Effect of metalaxyl resistance and cultivar resistance on control of Phytophthora nicotianae in tobacco. Plant Disease 86:362-366

Jain SS, Sharma SL and Juneja SL. 1961. Studies on buckeye rot of tomato- new to Himachal Pradesh. Indian Science Congress Association 50: 351-352

Kato M, Mizubuti ES, Goodwin SB and Fry WE. 1997. Sensitivity to protectant fungicides and pathogenic fitness of clonal lineages of Phytophthora infestans in the United States. Phytopathology 87:973-978

McKinney HH. 1923. Influence of soil temperature and moisture on infection of wheat seedlings by Helminthosporium sativum. Journal of Agricultural Research 26: $195-217$

Qu T, Shao Y, Csinos AS and Ji P. 2016. Sensitivity of Phytophthora nicotianae from tobacco to fluopicolide, mandipropamid and oxathiapiprolin. Plant Disease 100: 2119-2125

Sujkowaski LS, Fry BA, Power RJ, Goodwin SB, Peever TL, Hamlen RA and Fry WE. 1995. Sensitivity of Mexicon isolates of Phytophthora infestans to chlorothalonil, cymoxanil and metalaxyl. Plant Disease 79:1117-1120

Wang HC, Chen XJ, Cai LT and Cao Y. 2013. Race distribution and distribution of sensitivities to mefenoxam among isolates of Phytophthora parasitica var. nicotianae in Guizhou province of China. Crop Protection 52: 136-140

\section{How to cite this article:}

Gurpreet Kaur and Banyal, D.K. 2019. Sensitivity of Phytophthora nicotianae var. parasitica Causing Buckeye Rot of Tomato to Commonly Used Fungicides in Himachal Pradesh. Int.J.Curr.Microbiol.App.Sci. 8(08): 1198-1207. doi: https://doi.org/10.20546/ijcmas.2019.808.141 